\title{
Nutrition: more than the sum of its parts
}

\author{
The modern craze for dietary supplements is under increasing scrutiny, while biofortified crops look \\ promising in the quest to deliver nutrition in developing countries
}

\author{
Philip Hunter
}

M icronutrients are big business. Over the past century, scientists have established the ingredients of a healthy human diet, which should include very small amounts of 10 or so trace elements and 13 organic vitamins. The refinement, packaging and sale of these dietary 'supplements' has given rise to a vast industry, founded on the principle that a little more of what is good for you cannot be bad. This approach has even been successful for improving the health of people in regions in which micronutrients are scarce in the diet. However, evidence for the efficacy of micronutrient supplements in general, even to alleviate the effects of poverty, has been elusive.

\section{...scientific focus is therefore moving towards the engineering of food plants that contain trace elements and compounds in the right proportions and chemical forms}

At the same time, there is growing anecdotal evidence-now supported by nutritional research-that micronutrients function better when consumed as part of a balanced diet, rather than as pills. The exception is in specific cases of disease or deficiency, where micronutrient supplements can be effective. Yet, even here, it is becoming clear that increasing the micronutrient content of food crops to improve the whole diet offers greater improvements in quality of life and a reduction in mortality. The scientific focus is therefore moving towards the engineering of food plants that contain trace elements and compounds in the right proportions and chemical forms.

Those studying nutrition are also starting to take a more holistic approach. "I think that it is important to consider micronutrients as part of a network of bioactive constituents within the food and the organism," commented Jan Frank, group leader for bioavailability and bioactivity of micronutrients at the Institute of Biological Chemistry and Nutrition at Hohenheim University (Stuttgart, Germany). However, Frank admitted that doing so would require new scientific tools and a different emphasis in research. "Scientific experiments are most frequently designed in such a way that only a single parameter, or at the most a few parameters, are changed within an experimental setting."

Nevertheless, there is growing empirical evidence that several micronutrients act in combination rather than in isolation. According to Sabeeha Merchant, head of a research programme on trace-metal metabolism at the University of Los Angeles, California, "if some element B is present in excess, it can have an antagonistic impact on the assimilation of element A [...] One example is molybdenum, where excess amounts can result in reduced copper assimilation." This sort of deficiency has been observed in domestic and farm animals, especially ruminants (Spears, 2003), that depend on single sources of food. Humans with a varied diet are less likely to suffer from this problem, Merchant noted. Nevertheless, "if variation is reduced, as in the case of isolated communities, then there is the potential for toxicity or deficiency resulting from the presence of elements in the plants," Merchant said.
$\mathrm{T}$ he ability of micronutrients to antagonize each other could have important implications for human nutrition. For several decades, various interested parties have advised the public to 'take out insurance' against deficiency by swallowing more than the minimum recommended dose of key micronutrients. Furthermore, it has been suggested that taking excessively large doses of some micronutrients can protect against infection and disease. The Nobel-prize-winning chemist Linus Pauling could be charged with starting - or at least fuelling - this idea with his insistence that consuming large quantities of vitamin C afforded him extra protection against colds and flu late in his life (Pauling, 1976). He went on to suggest that such high doses might also protect against cancer and heart disease, increase lifespan and make people feel better, even when they are not actually ill (Pauling, 1986). These views have now been largely discredited. Frank commented that, "Overall, there is little evidence to suggest that the intake of micronutrients at concentrations beyond those achievable through a balanced diet may offer additional health benefits."

Another point is that some micronutrients are toxic at levels only a few times greater than the minimum recommended dose. A good example, according to Elizabeth Pilon-Smits, head of a research programme focusing on how plants metabolize trace elements and pollutants at Colorado State University, USA, is the element selenium. "For selenium, selenate and selenite are the most toxic forms," she said. "50-70 micrograms of selenium is needed per day for a human, and no more than 200 micrograms per day should be taken, 


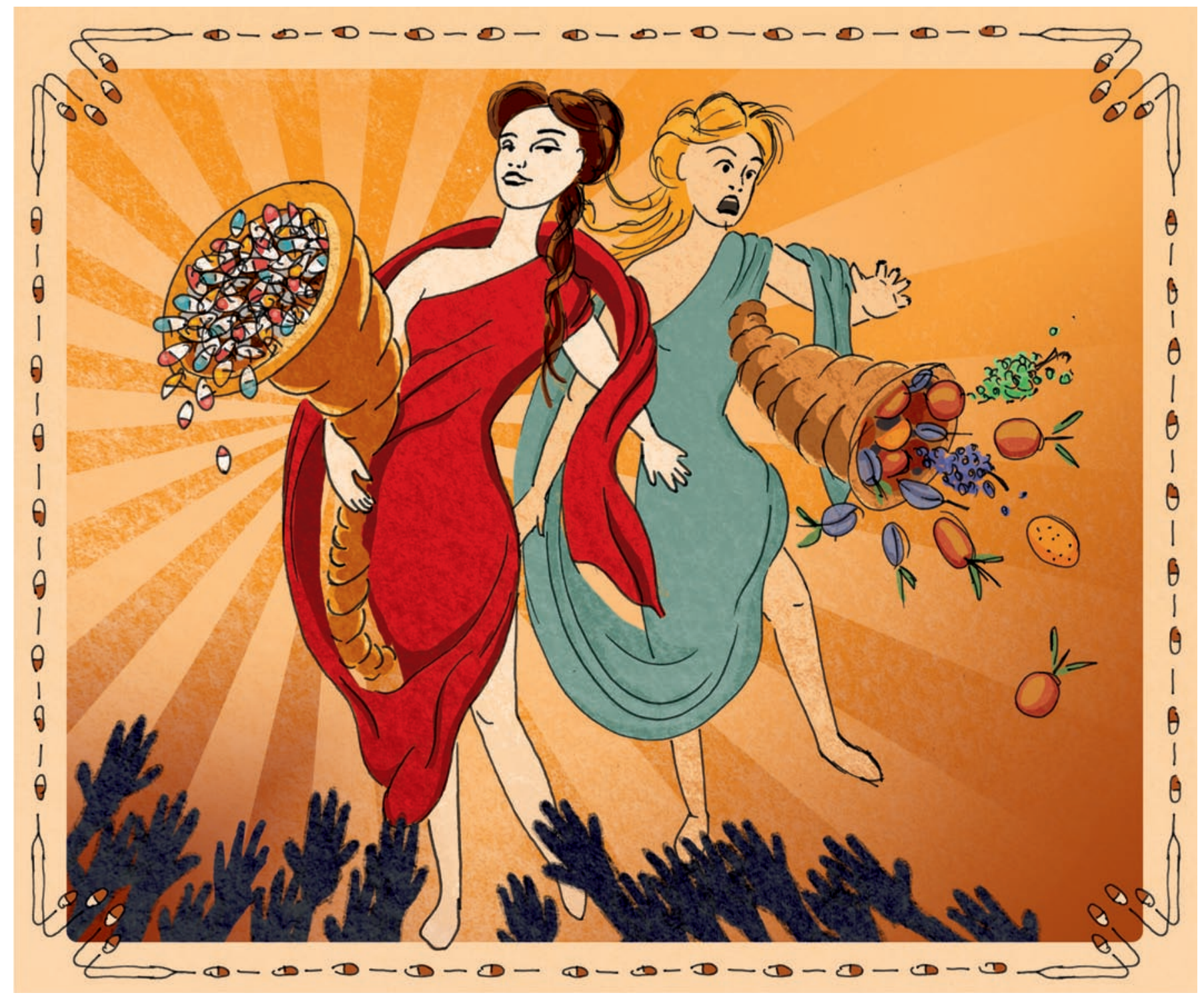

or it can result in chronic toxicity, so the window is very narrow."

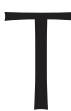

here is one exception to the general rule that a healthy diet provides sufficient quantities of the principal micronutrients. Vitamin D is unique among micronutrients in that its precursor is manufactured through a photosynthetic process that occurs on exposure of the skin to sunlight. However, this only produces adequate vitamin $\mathrm{D}$ when the sunlight is relatively strong, and diet alone is unable to make up the deficit during the autumn and winter months across much of the developed world. Partly for this reason, the recommended dietary intake of vitamin D was upgraded in November 2010 ...it has been suggested that taking excessively large doses of some micronutrients can protect against infection and disease

from $5 \mu \mathrm{g}$ to $15 \mu \mathrm{g}$ per day by the Institute of Medicine of the National Academies in the USA. However, it might be hard to achieve this level without the help of supplements.

"The most recent National Nutrition Survey II (Nationale Verzehrsstudie II) in Germany found that more than $88 \%$ of Germans did not even meet that recommended daily intake of vitamin D of $5 \mu \mathrm{g}, "$ Frank noted. "Consequently, it appears questionable whether the potentially higher requirements can be met by diet-especially in the absence of sufficient exposure to sunlightand consumption of vitamin D-fortified foods and/or dietary supplements may be necessary to maintain optimum health."

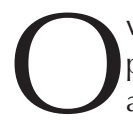
ver time, organisms and human populations can adapt to shortages of micronutrients, but this too has an inherent danger: chronic toxicity resulting from consumption of 'normal' levels of micronutrients. In such cases, levels that are safe for one group are toxic for another population, as has been observed in the case of Ronaldsway sheep, which live entirely on algae growing by the seashore on the Orkney Islands in the UK. These algae are deficient in copper, and the sheep have become highly sensitive to the element as 
a result. If they are moved to conventional grass-grazing pastures where they are able to consume-for them-an excess of copper, they suffer from oxidative stress (Haywood et al, 2005).

T he bigger picture is that the cocktail of micronutrients needed by all organisms is most effective when delivered through foods that contain other ingredients that aid absorption and metabolism. As Frank noted, it is almost common knowledge that vitamins soluble in lipids will only be absorbed efficiently in the presence of dietary fat. "In the context of our own research, we observed that simultaneously ingested phytochemicals, such as sesame lignans (present in sesame seeds and unrefined sesame oil) or alkylresorcinols (in wholegrain cereals, particularly rye) alter the metabolism and excretion of vitamin $\mathrm{E}^{\prime \prime}$ (Frank, 2005).

This leads to another dimension of the system, which is that micronutrients interact not only with each other, but also with clinical drugs. This has implications for the safety and efficacy of the latter, according to Frank. "For example, substances such as phytochemicals or micronutrients that affect phase I enzymes, may also alter the pharmacokinetics, the activity and ultimately the safety of drugs," he said.

\section{...the cocktail of micronutrients needed by all organisms is most effective when delivered through foods that contain other ingredients that aid absorption and metabolism}

Frank and his colleagues are studying the potential use of curcumin for the treatment and prevention of degenerative diseases including Alzheimer and some cancers. They are part of a three-year research network that started in August 2010, comprising five academic and five industry partners and supported by a $€ 1.5$ million grant from the German Federal Ministry of Education and Research. Curcumin is not an essential micronutrient; it is best known as the principal ingredient of tumeric-a spice for curries-and as a colouring agent. However, it also contains powerful antioxidants that might confer protection and act against several diseases.

Frank's research is seeking to address the problem that curcumin is poorly absorbed and rapidly excreted by most organisms. "We are investigating novel combinations of bioactive compounds as well as different formulations and food matrices (meaning complete foods incorporating many micronutrients as well as bulk nutrients such as protein) for their potential to enhance the absorption and retention of curcumin in the body," Frank explained. "We thus hope to identify new ways of improving the biological activities of curcumin, which may ultimately lead to the development of curcumin-containing functional foods." Such research is bridging the gap between nutrition and pharmacology.

$\mathrm{n}$ the immediate future, the greatest health benefits for many people in developing countries will either come from biofortified foods that contain normal levels of micronutrients or, in some cases, from single-nutrient supplements. One of the early breakthroughs came with research conducted by US ophthalmologist and epidemiologist Alfred Sommer, now working at the Johns Hopkins Bloomberg School of Public Health (Baltimore, MD, USA). During the 1970s and 1980s, Somner found that giving a high-dose vitamin A capsule to children deficient in the vitamin cut mortality levels by $34 \%$ (Sommer et al, 1986). This research led to several programmes in developing countries for the distribution of vitamin A.

Recently, it has become clear that still greater benefits will result from providing micronutrients through the biofortication of food crops that also provide staple calories and protein. Generating and distributing these crops is the long-term objective of HarvestPlus. This programme was set up in 2004 by the Consultative Group on International Agricultural Research (CGIAR) with funding from the Bill Gates Foundation, among other sources, to conduct research on biofortification of crops for developing countries. Its present focus is on three micronutrients recognized by the World Health Organization (WHO) to be deficient in many diets: iron, zinc and vitamin $\mathrm{A}$.

HarvestPlus has been working on a combination of plant breeding and genetics to develop crops that take up more of these micronutrients from the soil and make more of the nutrients bioavailable in the diet. In the case of zinc, fertilizer is applied to boost uptake by the plant in areas where zinc levels in the soil are low. Zinc deficiency itself is widespread, affecting around $25 \%$ of the world's population to some degree and causing a variety of problems relating to the impaired synthesis of proteins and DNA, including reduced resistance to infection and slow wound healing.

Although it has taken some time to establish that zinc uptake is increased by the consumption of biofortified plants, HarvestPlus is now convinced that it has firm evidence from field trials (Rosado et al, 2009) and is ready to launch zinc-fortified wheat and maize in India in 2013.

\section{...greater benefits will result from providing micronutrients through the biofortication of food crops that also provide staple calories and protein}

One interesting finding from the study is that the bioavailability of zinc depends on the levels of phytates in the crops, salts of phytic acid, which plants use as their primary source of stored phosphorus. Phytates bind (chelate) zinc ions, making them biologically unavailable in a high-phytate diet. HarvestPlus therefore had to take phytate levels into account when determining target zinc levels for its biofortified crops.

Biofortification for vitamin A has proven to be more straightforward: the first crop, a conventionally bred sweet potato with a high-vitamin-A content and an orange colour from the associated beta-carotene pigment, was launched in Uganda and Mozambique in 2007. The launch was followed by a study indicating that vitamin A was absorbed more effectively from the crop than from a supplement containing the same amount of vitamin A. People who ate the sweet potato accumulated the vitamin to higher levels, whereas consuming the supplements actually led to a slight decrease in stored levels over time. This suggested that people came to rely entirely on the supplements, although no explanation was given of why this should be (Tanumihardjo et al, 2008).

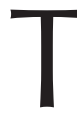
he work by HarvestPlus is already showing that vital micronutrient intake can be increased in developing countries through the use of biofortified crops. There is also plenty of evidence that diseases caused by deficiencies in a particular compound can be tackled with 
supplements, as in the case of vita$\min$ A. Yet, other studies suggest that the main impact of poverty extends beyond diet and cannot be addressed through improved nutrition alone. Such is the experience from Peru, where a study by the Tuberculosis Research Group at Imperial College (London, UK) has been evaluating whether dietary supplementation with vitamin $A$, vitamin $D$ and zinc reduces the risk of tuberculosis. The study recruited 800 healthy people in Peru who were at risk of contracting the disease for a randomized, blinded, placebo-controlled trial, which is approaching completion. The results are yet to be published, but preliminary data suggest that supplementation has failed to protect against tuberculosis, according to Carlton Evans, a research career development fellow in Clinical Tropical Medicine at the Wellcome Centre for Clinical Tropical Medicine at the College, and leader of the study.

\section{...although Evans agrees that nutrition is important, he argues that poverty is complex and that diseases cannot be cured or prevented just by distributing supplements}

"We have found very clearly that the main determinants of which of these people go on to get tuberculosis are very profoundly socioeconomic. The strongest single factor predicting tuberculosis is not having a cell phone." Factors such as overcrowding, despair, poor ventilation, stress and the other negative aspects of poverty seem to be at least as important as nutrition. Such conclusions are perhaps not too surprising. The same group began a second study in 2007 to ascertain whether socioeconomic interventions could reduce the incidence of tuberculosis. This work, called Innovative Socioeconomic Interventions Against Tuberculosis (ISIAT), recruited 2,078 people in 311 households in which there were newly diagnosed tuberculosis patients. The researchers conducted visits, held workshops, formed partnerships with existing organizations and provided low-level economic relief, such as by making small lines of credit available. The study is about to publish preliminary findings indicating that these measures can prevent tuberculosis more effectively than micronutrient supplementation.
Thus, although Evans agrees that nutrition is important, he argues that poverty is complex and that diseases cannot be cured or prevented just by distributing supplements. He also reiterated that sometimes giving supplements is exactly the wrong thing to do. "It has been shown, for example, that giving iron to people with malaria can cause them to die, because if someone is very frail, spicing up their immune system can be fatal."

The story of nutrition is complex, but a new, more complete picture is emerging. In regions in which micronutrient deficiency is rare, the focus of health professionals, nutrition experts and scientists is on producing and promoting foods containing appropriate forms and proportions of micronutrients. Although the convenience of popping a couple of pills with breakfast appeals to fast-paced modern societies, doing so is not usually an effective way to supplement the diet. In areas of poverty where deficiencies occur, crops that contain balanced proportions of micronutrients are effectivemore so than dietary supplements-but improved nutrition must be complemented by socioeconomic measures that address the other causes of disease. Finally, there is a new field of research emerging: what role do micronutrients have in drug effectiveness and safety? The proof of the micronutrient, as they say, will be in the eating.

\section{REFERENCES}

Frank J (2005) Beyond vitamin E supplementation: an alternative strategy to improve vitamin $\mathrm{E}$ status. J Plant Physiol 162: 834-843

Haywood S, Simpson DM, Ross G, Beynon RJ (2005) The greater susceptibility of North Ronaldsay sheep compared with Cambridge sheep to copper-induced oxidative stress, mitochondrial damage and hepatic stellate cell activation. J Comp Pathol 133: 114-127

Pauling L (1976) Vitamin C, the Common Cold and the Flu. San Francisco, CA, USA: WH Freeman

Pauling L (1986) How to Live Longer and Feel Better. New York, NY, USA: WH Freeman

Rosado JL et al (2009) The quantity of zinc absorbed by adult women is enhanced by biofortification. J Nutr 139: 1920-1925

Sommer A, Tarwotjo I, Djunaedi E, West KP Jr, Loeden AA, Tilden R, Mele L (1986) Impact of vitamin A supplementation on childhood mortality. A randomized controlled community trial. Lancet 24: 1169-1173

Spears JW (2003) Trace mineral bioavailability in ruminants. J Nutr 133: 1506S-1509S Tanumihardjo SA et al (2008) Food based approaches for ensuring adequate vitamin A nutrition. Comp Rev Food Sci Safety 7: 373-381

\section{Philip Hunter is a freelance journalist} in London, UK.

EMBO reports (2011) 12, 307-310.

doi:10.1038/embor.2011.42

\title{
Needles in a haystack
}

\author{
Genomic tools have become powerful tools for conservation biologists \\ to monitor the spread of invasive species
}

\section{Howard Wolinsky}

lot n the late 1960s, an Air France pilot and his family took a holiday in swampy Louisiana in the southeastern USA and were intrigued by the giant bullfrogs, Rana catesbeiana. The family introduced a dozen of the animals to a pond in the Bordeaux region of southwestern France, inadvertently starting a major invasion that affected thousands of lakes and creeks with devastating effects on the native fauna. "They eat everything and if you go to a pond where there are bullfrogs, there are no other amphibians [...] because bullfrogs prey on other animals or because they are spreading a disease-chytridiomycosisthat is absent from some European areas," said conservation biologist Francesco Ficetola of the Department of Environmental Sciences at the University of MilanoBicocca in Italy. "There was nothing like them in Europe," he added.

As part of his postdoctoral research, Ficetola studied the frog invasion in Europe at the Universite de Savoie and the University of Grenoble in France. To monitor their distribution, he pioneered the use of environmental DNA (eDNA) to detect $R$. catesbeiana without observing the 